\title{
CRECIMIENTO Y RESPUESTAS FISIOLÓGICAS DE Phaseolus spp. EN CONDICIONES DE SALINIDAD
}

\section{GROWTH AND PHYSIOLOGICAL RESPONSES OF Phaseolus spp. UNDER SALINITY}

\author{
Leobardo Bahena Betancourt ${ }^{1}$, Lourdes Macías Rodríguez², Rodolfo López Gómez ${ }^{2}$ \\ y Jeannette S. Bayuelo Jiménez ${ }^{1^{*}}$
}

${ }^{1}$ Instituto de Investigaciones Agropecuarias y Forestales e ${ }^{2}$ Instituto de Investigaciones Químico Biológicas, Universidad Michoacana de San Nicolás de
Hidalgo. Morelia, Michoacán. Km. 9.5 Carretera Morelia-Zinapécuaro. 58880, Tarímbaro, Michoacán, México. Tel. (01) 443 2958324. *Autor para correspondencia (jsbayuelo@hotmail.com)

\section{RESUMEN}

Se estudió el comportamiento de variables fisiológicas e índices de crecimiento en especies de Phaseolus en condiciones de salinidad. El experimento se realizó en solución nutritiva en condiciones de invernadero, con cuatro niveles de salinidad $(0,30,60$, and $90 \mathrm{mM}$ $\mathrm{NaCl})$. Durante $20 \mathrm{~d}$ de iniciado el tratamiento salino, se analizó la tasa de crecimiento relativo (TCR; $\mathrm{g} \mathrm{g}^{-1} \mathrm{~d}^{-1}$ ), tasa de asimilación neta (TAN; $\mathrm{g} \mathrm{m}^{-2}$ día $\left.^{-1}\right)$, razón de área foliar (RAF; $\mathrm{m}^{2} \mathrm{~g}^{-1}$ ), área foliar específica (AFE; $\mathrm{m}^{2} \mathrm{~g}^{-1}$ ) y relación de peso foliar (RPF; $\mathrm{g} \mathrm{g}^{-1}$ ). El incremento de salinidad redujo la TCR, TAN, RAF y AFE, pero no afectó la RPF. En todas las especies, excepto en $P$. filiformis, la TCR correlacionó significativamente con TAN y no con RAF, lo que indica que la eficiencia fotosintética en un factor determinante en las diferencias entre especies. En P. filiformis, la TCR, TAN y RAF se mantuvieron inalterables aún en la mayor concentración salina. En todas especies, excepto en $\boldsymbol{P}$. filiformis, el incremento de salinidad disminuyó progresivamente la conductancia estomática y la tasa de asimilación de $\mathrm{CO}_{2}$. Los potenciales hídrico y osmótico disminuyeron conforme se intensificó el agobio salino, pero el ajuste osmótico permitió que la turgencia celular se mantuviera o aumentara. La salinidad provocó incremento significativo de carbohidratos totales en hojas y tallos, pero no en raíces; tal acumulación de carbohidratos se relacionó con el mantenimiento de turgencia en etapas iniciales del agobio. Se confirmó que $\boldsymbol{P}$. filiformis es una especie tolerante a salinidad, tolerancia que está regulada por un adecuado control estomático y eficiencia fotosintética a través de ajuste osmótico.

Palabras clave: Phaseolus spp, crecimiento, relaciones hídricas, carbohidratos totales, tolerancia a salinidad.

\section{SUMMARY}

To study the behavior of physiological parameters and plant growth analysis under salt stress, Phaseolus species were grown under greenhouse conditions, in nutrient solution at four different salinity levels $(0,30,60$, and $90 \mathrm{mM} \mathrm{NaCl})$. Relative growth rate (RGR; $\mathrm{g} \mathrm{g}^{-1} \mathrm{~d}^{-1}$ ), net assimilation rate (NAR; $\mathrm{g} \mathrm{m}^{-2} \mathrm{~d}^{-1}$ ), leaf area ratio $\left(L A R ; m^{2} \mathbf{g}^{-1}\right)$, specific leaf area (SLA; $\mathrm{m}^{2} \mathrm{~g}^{-1}$ ) and leaf weight ratio (LWR; $\mathrm{g} \mathrm{g}^{-1}$ ) were calculated for $20 \mathrm{~d}$. The increase in salinity significantly reduced RGR, NAR, LAR and SLA, without affecting LWR. In all species, except in $P$. filiformis, NAR, but not LAR, was significantly correlated with RGR, thus indicating that NAR is an important factor underlying the salinity-induced differences among species. In $\boldsymbol{P}$. filiformis neither NAR nor LAR were affected by increased salinity, and consequently RGR was maintained. Increasing salinity progressively decreased the stomatal conductance. The $\mathrm{CO}_{2}$ assimilation rate decreased gradually with salinity, but significant reductions were showed only at the highest salt levels $(90 \mathrm{mM} \mathrm{NaCl})$. In $P$. filiformis, however, neither stomatal conductance nor $\mathrm{CO}_{2}$ assimilation were affected by salt stress. Leaf water and osmotic potentials declined significantly as stress conditions intensified, but osmotic adjustment permitted the maintenance of positive turgor throughout the growth period. Salinity increased the concentration of total carbohydrates in leaves and stems; this carbohydrate accumulation was associated to the osmotic adjustment at the initiation of the saline period. Salt tolerance in $\boldsymbol{P}$. filiformis is associated to a better stomatal control and photosynthetic efficiency through osmotic adjustment.

Index words: Phaseolus spp, plant growth, water relations, total carbohydrates, salinity tolerance.

\section{INTRODUCCIÓN}

Se estima que $20 \%$ de la superficie cultivable actual y aproximadamente la mitad de las áreas irrigadas a nivel mundial se encuentran afectadas por salinidad (FAO, 2005). En México, el frijol común (Phaseolus vulgaris L.) se cultiva principalmente en ambientes semitropicales, planicies semiáridas y en áreas irrigadas (Broughton et al., 2003). Se estima que $30 \%$ de las áreas irrigadas en México está afectado por salinidad y en áreas marginadas el problema es aún más severo (FAO, 2005). La salinidad afecta principalmente al metabolismo de la planta y reduce su crecimiento y productividad por efecto de potenciales hídricos reducidos, toxicidad iónica y desbalance nutrimental (Munns, 2002; Ruiz et al., 1997). La capacidad asimilatoria y el estado hídrico de la planta son procesos fisiológicos sensibles a la salinidad, además de ser factores determinantes en el crecimiento vegetal (Cramer et al., 1994). 
El estrés hídrico inducido por salinidad afecta el crecimiento de la planta a través de alteraciones en la división celular (Yeo et al., 1985), fotosíntesis (BayueloJiménez et al., 2003; Martínez-Ballesta et al., 2004), procesos bioquímicos (Plaut et al., 1990) y transpiración (Serraj y Sinclair, 2002). La regulación osmótica celular -mecanismo fisiológico que contrarresta el estrés osmótico causado por exceso de sales en el medio-, contribuye al mantenimiento de la absorción de agua y turgencia celular, y, consecuentemente, de otros procesos fisiológicos (Serraj y Sinclair, 2002). La regulación osmótica en plantas sometidas a estrés salino puede ocurrir por acumulación de solutos orgánicos compatibles (carbohidratos solubles, aminoácidos, prolina y betaína) (Hasewaga et al., 2000), de iones inorgánicos o de ambos (Stepien y Klobus, 2006). En especies cultivadas (glicófitas), el principal mecanismo de tolerancia es a través de exclusión de iones. Sin embargo, a bajas concentraciones de $\mathrm{NaCl}$ la inclusión de iones $\mathrm{Na}^{+}$y $\mathrm{Cl}^{-}$puede favorecer el ajuste osmótico (Munn, 2002).

El frijol común es una especie sensible a la salinidad que reduce su rendimiento ( $>50 \%$ ) a una conductividad eléctrica de saturación del extracto del suelo $(\mathrm{CE}) \geq$ a 2 $\mathrm{dS} \mathrm{m}{ }^{-1}$, equivalente a $20 \mathrm{mM} \mathrm{NaCl}$ (Läuchli, 1984). Su tolerancia a la salinidad es muy limitada y se encuentra asociada con una tasa reducida de absorción y transporte de $\mathrm{Na}^{+}$al vástago (Läuchli, 1984). Además, su crecimiento es afectado por la acumulación de $\mathrm{Cl}^{-}$en las hojas y por la alteración del estado nutrimental y de las relaciones hídricas (Bayuelo-Jiménez et al., 2003).

Por el contrario, en especies silvestres de Phaseolus existe una valiosa fuente de genes para tolerancia a salinidad, particularmente en $P$. angustissimus, $P$. acutifolius, $P$. filiformis, $P$. lunatus y $P$. vulgaris (Bayuelo-Jiménez et $a l ., 2002)$. Dicha tolerancia se asocia con mayor velocidad de germinación, menor reducción del crecimiento relativo y mayor relación raíz:vástago (Bayuelo-Jiménez et $a l .$, 2002). Asimismo, estas especies poseen un mecanismo de exclusión de $\mathrm{Na}^{+}$en raíz y ajuste osmótico para mantenimiento de la turgencia mediante la acumulación de $\mathrm{Cl}^{-}$(Bayuelo-Jiménez, 2003). No obstante, la información relacionada con los índices fisiológicos que determinan la tolerancia en Phaseolus y su relación con diferencias genotípicas es reducida. Así, el objetivo de este estudio fue determinar el comportamiento de características fisiológicas e índices de crecimiento en respuesta a la salinidad, en especies de Phaseolus.

\section{MATERIALES Y MÉTODOS}

La presente investigación se hizo en condiciones de invernadero y cultivo en solución nutritiva, de abril a ju- lio del 2005, a una temperatura promedio de $35^{\circ} \mathrm{C} \pm 2$ con una máxima de $38^{\circ} \mathrm{C}$ y mínima de $15^{\circ} \mathrm{C}$. La humedad relativa varió de 60 a $70 \%$. El experimento incluyó cuatro tratamientos, tres correspondientes al estrés salino $(30,60$ y $90 \mathrm{mM} \mathrm{NaCl})$ y el testigo $(0 \mathrm{mM} \mathrm{NaCl})$, combinados con cuatro especies de Phaseolus: dos especies silvestres [P. acutifolius Gray (G40169) y P. filiformis Benham (PI535309)] y dos cultivadas: [P. acutifolius (G40142) у P. vulgaris L. (G04017)]. Las dos especies silvestres se eligieron por su adaptación a zonas áridas y por su grado de regulación iónica y tolerancia a salinidad previamente identificadas (Bayuelo-Jiménez et al., 2002). Phaseolus acutifolius silvestre excluye $\mathrm{Na}^{+}$y acumula $\mathrm{Cl}^{-}$ (sensible); $P$. filiformis excluye $\mathrm{Na}^{+}$e incluye $\mathrm{K}^{+}$(tolerante); $P$. acutifolius cultivado excluye $\mathrm{Na}^{+} \mathrm{y} \mathrm{Cl}^{-}$(tolerante) y $P$. vulgaris excluye $\mathrm{Na}^{+}$e incluye $\mathrm{Cl}^{-}$(sensible). Antes de establecer el cultivo en solución, las semillas se esterilizaron con $2.5 \mathrm{~g} \mathrm{~L}^{-1}$ de hipoclorito de sodio $5 \%(\mathrm{v} / \mathrm{v})$ y agua desionizada; luego se escarificaron mecánicamente y germinaron en una incubadora a $25{ }^{\circ} \mathrm{C}$ en papel de germinación (Anchor Paper Co., St. Paul, MN, USA) humedecido con una solución $0.5 \mathrm{mM}$ de $\mathrm{CaSO}_{4}$. Las plántulas de $7 \mathrm{~d}$ y de tamaño uniforme se transfirieron a tanques aireados (volumen de $100 \mathrm{~L}$ ), los cuales contenían la solución nutritiva (Epstein, 1972).

La composición de la solución en mM fue de $6 \mathrm{KNO}_{3}$, $4 \mathrm{Ca}\left(\mathrm{NO}_{3}\right)_{2} 1 \mathrm{MgSO}_{4} 1 \mathrm{NH}_{4} \mathrm{H}_{2} \mathrm{PO}_{4}, 0.025 \mathrm{Fe}-\mathrm{EDTA}, 0.05$ $\mathrm{KCL}, 0.025 \mathrm{H}_{3} \mathrm{BO}_{3}, 0.002 \mathrm{MnSO}_{4}, 0.005 \mathrm{CuSO}_{4}, \mathrm{y}$ $0.0005\left(\mathrm{NH}_{4}\right) 6 \mathrm{Mo}_{7} .4 \mathrm{H}_{2} \mathrm{O}$. El pH de la solución se ajustó diariamente a 6-6.5 con una solución amortiguadora de $\mathrm{KOH} 1 \mathrm{~N}$. Todas las plantas se mantuvieron en esta solución nutritiva hasta la emergencia de la primera hoja trifoliolada ( $7 \mathrm{~d}$ después del transplante), momento en el cual se inició la aplicación del tratamiento salino. Se hicieron aplicaciones diarias nocturnas de $30 \mathrm{mM} \mathrm{NaCl}$ y $3 \mathrm{mM}$ de $\mathrm{CaCl}_{2}$ hasta alcanzar una concentración final de 30,60 y $90 \mathrm{mM} \mathrm{NaCl}$ y 3,6 y $9 \mathrm{mM}$ de $\mathrm{CaCl}_{2}$. La adición de $\mathrm{CaCl}_{2}$ al medio nutritivo fue una medida preventiva para evitar el choque osmótico al inicio de la aplicación de los tratamientos salinos.

Las plantas se cosecharon a los 10 y 20 d después de aplicar el tratamiento salino. Se cuantificó el número de hojas y el área foliar por planta con planímetro portátil modelo L1-300 (LI-COR, Lincoln, NE, USA). Las hojas, tallo y raíz se deshidrataron en una estufa a $65{ }^{\circ} \mathrm{C}$ por 96 h, para luego medir el peso seco. Los índices del análisis de crecimiento se calcularon de acuerdo con Hunt (1990). La tasa relativa de crecimiento (TCR, $\mathrm{g} \mathrm{g}^{-1} \mathrm{~d}^{-1}$ ) se define como el incremento de materia seca por unidad de materia seca presente, por unidad de tiempo. Los componentes de crecimiento incluyeron la tasa de asimilación neta media (TAN, $\mathrm{g} \mathrm{m}^{-2} \mathrm{~d}^{-1}$ ) y la razón de área foliar $\left(\mathrm{RAF}, \mathrm{m}^{2} \mathrm{~g}^{-1}\right.$ ). 
TAN se calculó a partir de la relación de incremento de materia seca total por unidad de área foliar por día (RAF) y ésta se calculó como la relación entre el área foliar total y el peso de la materia seca total de la planta. La razón de peso foliar (RPF, $\mathrm{g} \mathrm{g}^{-1}$ ) se determinó como el cociente entre la materia seca total de la hoja y de la planta. El área foliar específica (AFE, $\mathrm{m}^{2} \mathrm{~g}^{-1}$ ) se refiere al área foliar por unidad de peso foliar.

Las mediciones de asimilación neta de $\mathrm{CO}_{2}$ y conductancia estomática se hicieron a los $20 \mathrm{~d}$ después del inicio de la aplicación de los tratamientos salinos, con un analizador de gases al infrarrojo LI-COR 6400 (LI-COR, Lincoln, NE, USA). Tales mediciones se hicieron en el foliolo central de la quinta hoja trifoliolada, la cual se introdujo en una cámara foliar con temperatura y aireación controlada $\left(6 \mathrm{~cm}^{2}\right)$. La fotosíntesis neta se midió a una presión parcial externa de $34 \mathrm{MPa}\left(340 \mu \mathrm{mol} \mathrm{CO}_{2} \mathrm{~mol}^{-1}\right.$ aire), densidad de presión de vapor de $1.8 \mathrm{kPa}$ y una densidad de flujo fotónico de $1200 \mu \mathrm{mol} \mathrm{m} \mathrm{m}^{-2} \mathrm{~s}^{-1}$.

Las mediciones del potencial hídrico $\left(\Psi_{\mathrm{w}}\right)$ se hicieron entre las 3:00 y 5:00 am y 19 d después del inicio de la aplicación de los tratamientos salinos, en las mismas hojas en donde se midió la asimilación neta de $\mathrm{CO}_{2}$, con una cámara de presión Modelo 3000 (Soilmosture, Santa Barbara, CA, USA) (Scholander et al., 1965). El potencial osmótico $(\Psi \pi)$ se calculó a partir de la medición de la osmolaridad de las hojas previamente evaluadas para el potencial hídrico. El tejido foliar se almacenó en nitrógeno líquido y posteriormente se maceró con un homogenizador portátil, y la muestra se centrifugó en una microcentrífuga Eppendorf por 5 min a $2000 \times \mathrm{g}$. Se utilizó un volumen de $10 \mu \mathrm{mol}$ del sobrenadante y se midió la osmolaridad con un osmómetro Wescor 5520XR, (Logan, UT, USA). Las lecturas de osmolaridad se convirtieron a unidades de presión por medio de la ecuación de Van' Hoff $(\pi=-\mathrm{RT})$, donde $c$ es la osmolaridad (mosmol kg-1), R es la constante de los gases y $\mathrm{T}$ es la temperatura $(\mathrm{K})$ (Nobel, 1991). El potencial de turgencia o presión $\left(\Psi_{\mathrm{p}}\right)$ se determinó por diferencia de presiones con la ecuación: $\Psi_{\mathrm{p}}$ $=\Psi_{\mathrm{w}-} \Psi \pi$.

La extracción de los azúcares de hoja, tallo y raíz se hizo mediante la metodología descrita por Ojeda-Real $e t$ al. (2008). A $2 \mathrm{~g}$ de tejido previamente congelado en nitrógeno líquido y almacenado a $-80{ }^{\circ} \mathrm{C}$ se les adicionaron $20 \mathrm{~mL}$ de etanol $80 \%$ para su extracción. El tejido se incubó a $60{ }^{\circ} \mathrm{C}$ con agitación constante por $12 \mathrm{~h}$; transcurrido ese tiempo se filtró al vacío, con papel filtro Watman de $3 \mathrm{~mm}$. Al residuo obtenido se le agregó nuevamente $20 \mathrm{~mL}$ de etanol $80 \%$, se agitó durante $3 \mathrm{~h}$ y se volvió a filtrar. Los extractos se unieron y se adicionó carbón activado para eliminar los pigmentos; posterior- mente la muestra se concentró en un rotavapor a $65^{\circ} \mathrm{C}$ y se resuspendió en $5 \mathrm{~mL}$ de etanol $80 \%$. Para la determinación de azúcares totales se tomó $1 \mathrm{~mL}$ del extracto etanólico y se diluyó en un volumen de $25 \mathrm{~mL}$. De esta dilución se tomaron $2 \mathrm{~mL}$ para realizar la determinación mediante el método de Dubois et al. (1956). Este análisis se efectuó por triplicado.

Se empleó un diseño experimental de bloques al azar con un arreglo de tratamientos de parcelas divididas y cuatro repeticiones por especie. Los niveles de $\mathrm{NaCl}$ correspondieron a la parcela principal y las especies distribuidas aleatoriamente dentro de cada parcela, correspondieron a las sub-parcelas. Con el objeto de verificar las diferencias significativas entre especies en cada variable considerada, se hicieron pruebas de $\mathrm{F}$ mediante procedimiento GLM (SAS, Institute, 1995). Se utilizó la prueba protegida DMS (diferencia mínima significativa) de Fisher para comparar las diferencias identificadas con el análisis de varianza (SAS, Institute, 1995).

\section{RESULTADOS Y DISCUSIÓN}

\section{Biomasa aérea y radical}

Los tratamientos salinos tuvieron un efecto significativo en el número de hojas, área foliar, peso seco de hoja, tallo y raíz (Cuadro 1). Asimismo, las diferencias entre especies, tratamientos salinos y las interacciones para todas las variables fueron significativas. En todas las especies, excepto en $P$. filiformis, la salinidad redujo la acumulación de materia seca del vástago (de 22 a $44 \%$ ) y de la raíz (de 22 a $62 \%$ ). En $P$. filiformis se observó un incremento de materia seca del vástago a $30 \mathrm{mM} \mathrm{NaCl}$ (33\%) y una reducción de sólo $22 \%$ a $90 \mathrm{mM} \mathrm{NaCl}$. El número de hojas disminuyó significativamente al aumentar la concentración de $\mathrm{NaCl}$, lo que promovió una disminución del área foliar en todas las especies, excepto en $P$. filiformis. A los $20 \mathrm{~d}$ de exposición al agobio salino, el área foliar total del resto de las especies disminuyó de 28 a $45 \%$ en $60 \mathrm{mM} \mathrm{NaCl}$ y de 44 a $60 \%$ en $90 \mathrm{mM} \mathrm{NaCl}$.

\section{Índices de crecimiento}

El agobio salino disminuyó significativamente la tasa de crecimiento relativo (TCR). Hubo diferencias significativas $(P \geq 0.01)$ entre tratamientos de salinidad y entre especies; sin embargo, la interacción entre estos dos factores no fue significativa (Cuadro 2). Entre especies, la TCR del tratamiento testigo varío de 0.10 a $0.17 \mathrm{~g} \mathrm{~g}^{-1} \mathrm{~d}^{-1}$, mientras que en las plantas sometidas a los tratamientos de salinidad varió de 0.07 a $0.14 \mathrm{~g} \mathrm{~g}^{-1} \mathrm{~d}^{-1}$ (Figura 1A); en $P$. acutifolius silvestre (G40169) y cultivada (G40142) y en $P$. vulgaris cultivada (G04017), la TCR disminuyó al 
incrementar el agobio salino mientras que en $P$. filiformis silvestre (PI535309) la TCR no fue afectada por la salinidad.

La tasa de asimilación neta (TAN) disminuyó significativamente al aumentar la concentración de $\mathrm{NaCl}$, pero las diferencias entre especies y la interacción no fueron significativas (Cuadro 2). La reducción de TAN siguió una tendencia similar a la TCR, excepto que la reducción de TAN fue mayor a $90 \mathrm{mM} \mathrm{NaCl}$ en todas las especies, menos en $P$. filiformis cuya TAN se mantuvo similar al testigo en todos los tratamientos salinos (Figura 1B). El agobio salino disminuyó significativamente la razón de área foliar (RAF), y las diferencias entre especies y la interacción entre $\mathrm{NaCl}$ y especies fue significativa (Cuadro 2). En las especies cultivadas [P. acutifolius (G40142) y $P$. vulgaris (G04017)], la RAF disminuyó a mayor concentración salina pero se mantuvo inalterable en las especies silvestres (Figura 1C). La razón de peso foliar (RPF) se mantuvo constante en todas los tratamientos salinos y especies, mientras que el área foliar específica (AFE) disminuyó significativamente a mayor concentración de $\mathrm{NaCl}$ solamente en las especies cultivadas, pero no en la especie silvestre $P$. acutifolius (Figuras 1D-E).

Cuadro 1. Efecto del $\mathrm{NaCl}$ en el número de hojas, área foliar, peso seco de hoja, tallo y raíz de Phaseolus spp.

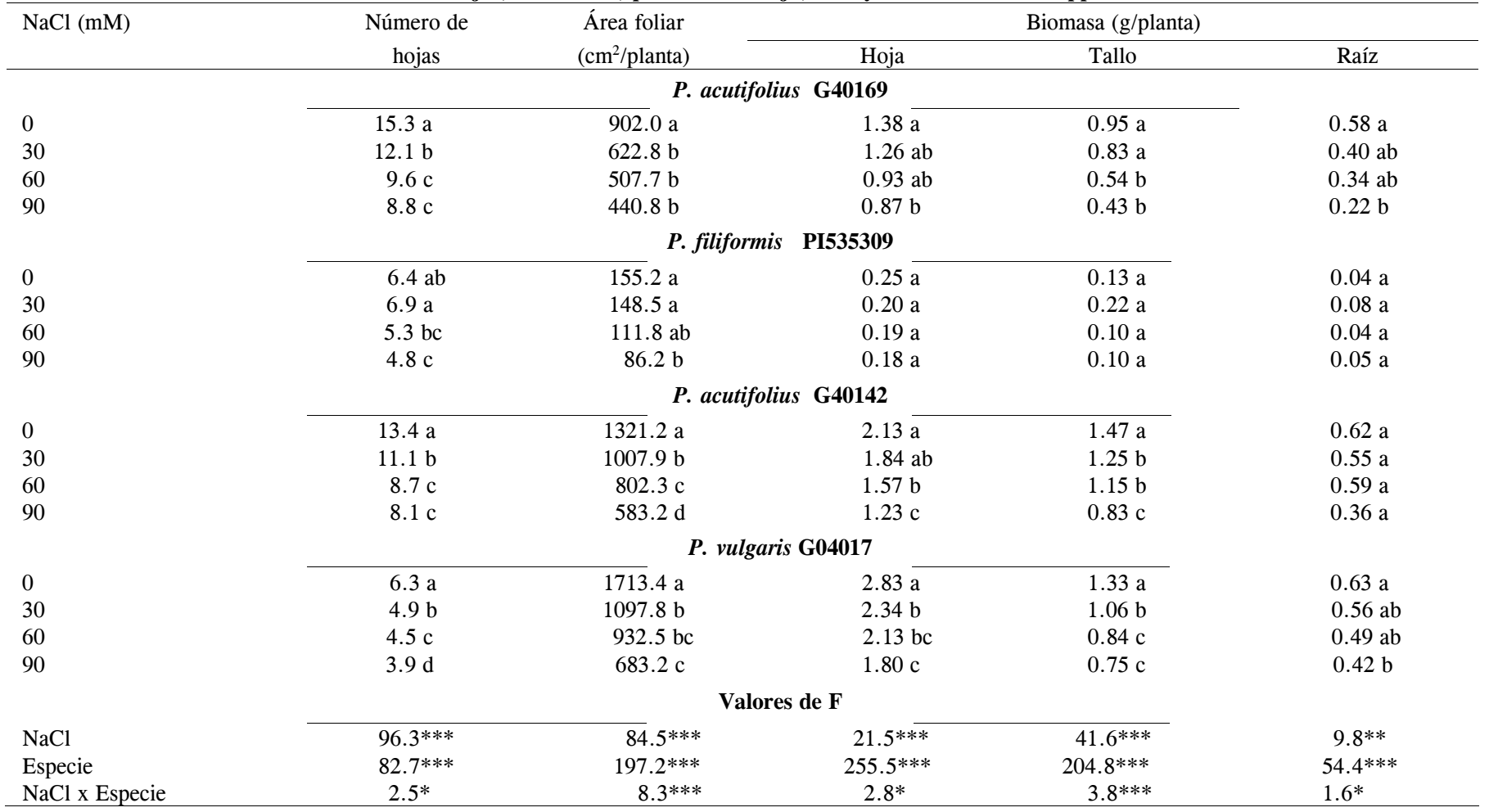

Las medias son valores promedio de cuatro repeticiones después de $20 \mathrm{~d}$ de exposición a $\mathrm{NaCl}$. Prueba DMS (diferencia mínima significativa) protegida de Fisher a $\mathrm{P} \leq 0.05$. *,**,*** Significativo a $\mathrm{P} \leq 0.05, \mathrm{P} \leq 0.01, \mathrm{P} \leq 0.001$, respectivamente.

Cuadro 2. Cuadrados medios y su significancia para la tasa relativa de crecimiento (TCR), tasa de asimilación neta (TAN), razón de área foliar (RAF), relación de peso foliar (RPF), área foliar específica (AFE), tasa de asimilación de $\mathrm{CO}_{2}$ (A), conductancia estomática (gs), potencial hídrico $\left(\Psi_{\mathrm{w}}\right)$, potencial osmótico $\left(\Psi_{\Pi}\right)$, potencial de turgencia $\left(\Psi_{\mathrm{t}}\right)$, carbohidratos totales de hoja $\left(\mathrm{CHO}_{\mathrm{h}}\right)$, tallo $\left(\mathrm{CHO}_{\mathrm{t}}\right)$ y raíz $\left(\mathrm{CHO}_{\mathrm{r}}\right)$ de Phaseolus spp., después de $20 \mathrm{~d}$ de estrés salino.

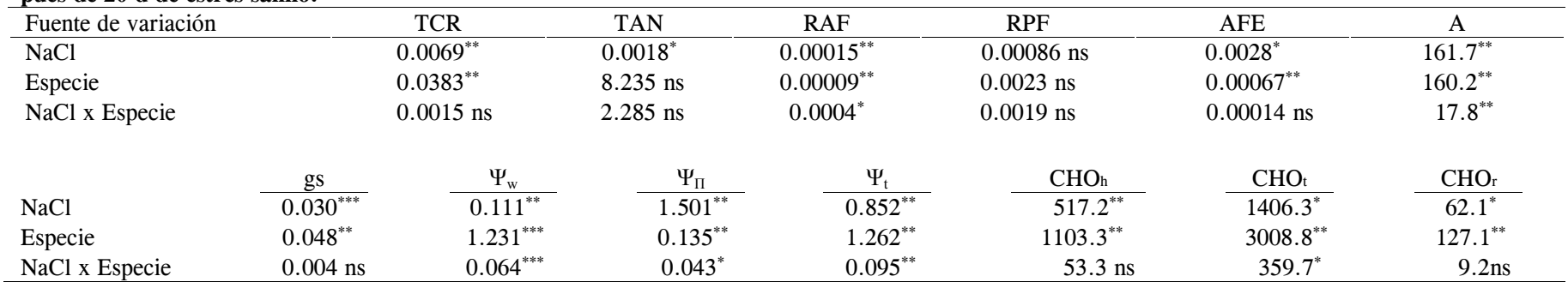

$*$, ** Significativo a $\mathrm{P} \leq 0.05, \mathrm{P} \leq 0.01$, respectivamente; $\mathrm{ns}=$ no significativo. 

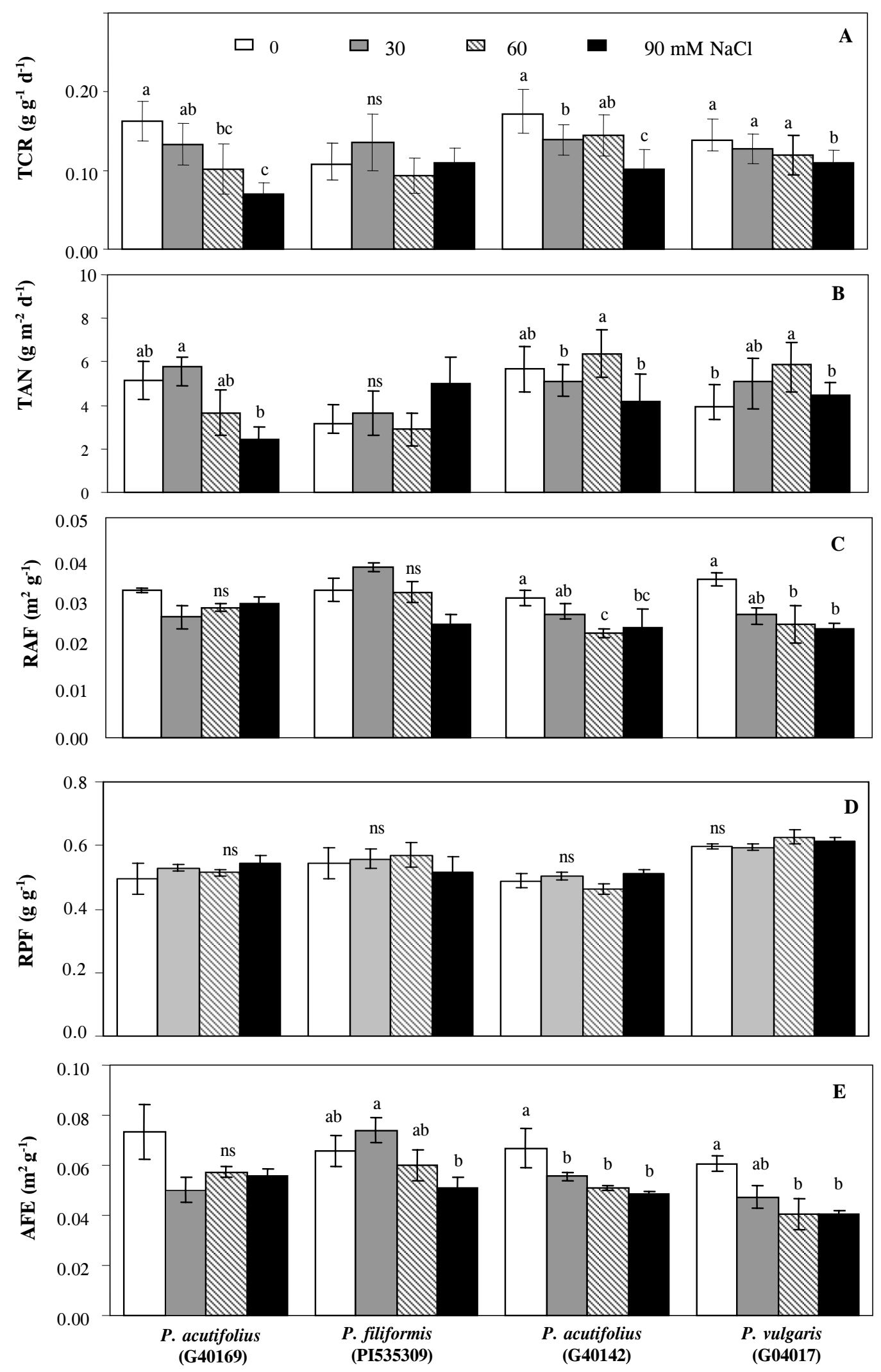

Figura 1. Efecto del NaCl en la tasa de crecimiento relativo TCR (A), tasa de asimilación neta TAN (B), razón de área foliar RAF (C), razón de peso foliar RPF (D) y área foliar específica AFE (E) de Phaseolus, después de $20 \mathrm{~d}$ de exposición a salinidad. Las letras sobre las barras indican diferencias significativas (Prueba DMS protegida de Fisher, $P \leq 0.05$ ). 


\section{Fotosíntesis y conductancia estomática}

La salinidad afectó significativamente la conductancia estomática y la asimilación de $\mathrm{CO}_{2}$. Sin embargo, sólo en el caso de la asimilación de $\mathrm{CO}_{2}$ la interacción salinidad $\mathrm{x}$ especie resultó significativa (Cuadro 2). La salinidad redujo la conductancia estomática y la asimilación de $\mathrm{CO}_{2}$ a medida que aumentó la concentración salina, con reducciones significativas a 60 y $90 \mathrm{mM} \mathrm{NaCl}$ (Figuras 2A-D). En condiciones salinas, la conductancia estomática de $P$. vulgaris cultivada fue menor que la de las especies restantes, con una reducción de $26 \%$ en $90 \mathrm{mM} \mathrm{NaCl}$ (Figura 2D). Por el contrario, en $P$. filiformis hubo reducción de la conductancia estomática al acentuarse el agobio salino (Figura $\mathrm{B}$ ). La asimilación de $\mathrm{CO}_{2}$ disminuyó significativamente en las especies silvestres y cultivadas de $P$. $a c u$ tifolius en $60 \mathrm{mM} \mathrm{NaCl}$ (Figuras A y C), mientras que en $P$. vulgaris disminuyó sólo en $90 \mathrm{mM} \mathrm{NaCl}$ (Figura 2D). La asimilación de $\mathrm{CO}_{2}$ de $P$. filiformis, por el contrario, se mantuvo invariable en todos los tratamientos salinos (Figura 2B); en el resto de las especies se registró un incremento de 15 a $40 \%$ en la tasa fotosintética, en $30 \mathrm{mM}$
$\mathrm{NaCl}$. Posteriormente, las reducciones en la fotosíntesis aumentaron en función del nivel de salinidad.

\section{Relaciones hídricas}

La salinidad afectó significativamente los potenciales de turgencia, hídrico y osmótico (Cuadro 2). Las diferencias entre especies y sus interacciones fueron significativas en todos los tratamientos salinos. En general, el potencial hídrico de las especies silvestres de $P$. acutifolius G40169 y P. filiformis PI535309 fue mayor en todos los tratamientos salinos que en las especies cultivadas (Figura 3B). La salinidad disminuyó el potencial osmótico en todas las especies, pero las diferencias entre éstas sólo se observaron a $90 \mathrm{mM}$ de $\mathrm{NaCl}$. P. filiformis fue la única especie que mantuvo el mayor potencial osmótico (-1.13 $\mathrm{MPa}$ ) en $90 \mathrm{mM}$ de $\mathrm{NaCl}$ (Figura 3C), mientras que en el resto, el potencial osmótico osciló entre -1.25 y -1.44 MPa (Figura 3C). El potencial de turgencia se mantuvo constante hasta los $30 \mathrm{mM} \mathrm{NaCl}$ y aumentó significativamente a 60 y $90 \mathrm{mM}$ de $\mathrm{NaCl}$ en todos las especies excepto en $P$. vulgaris cultivado (Figura 3A).

\section{P. acutifolius (G40169)}

\section{P. filiformis (P1535309)}

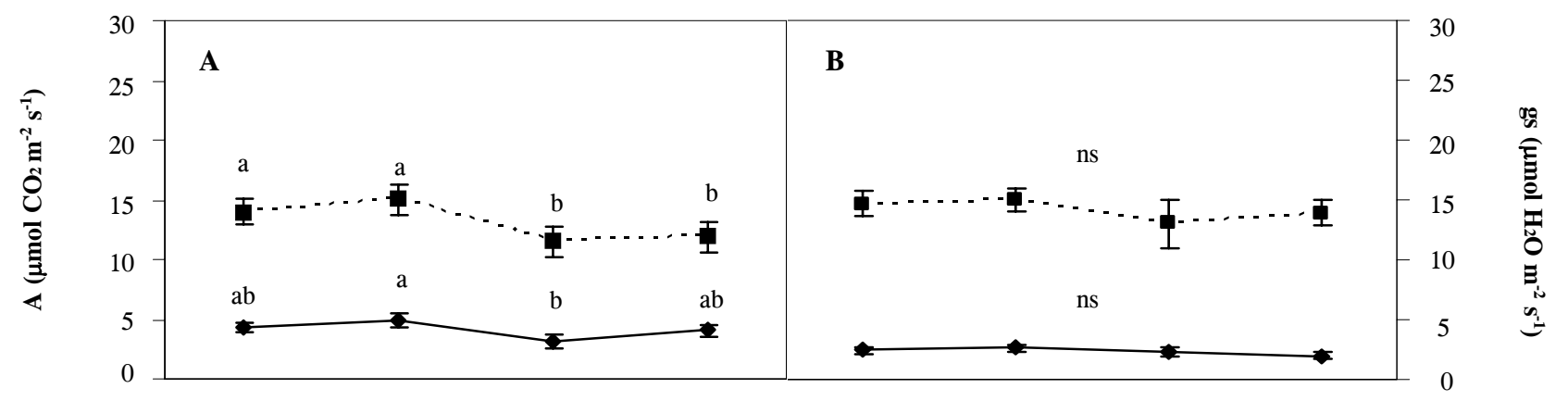

P. acutifolius (G40112)

P. vulgaris (G04017)

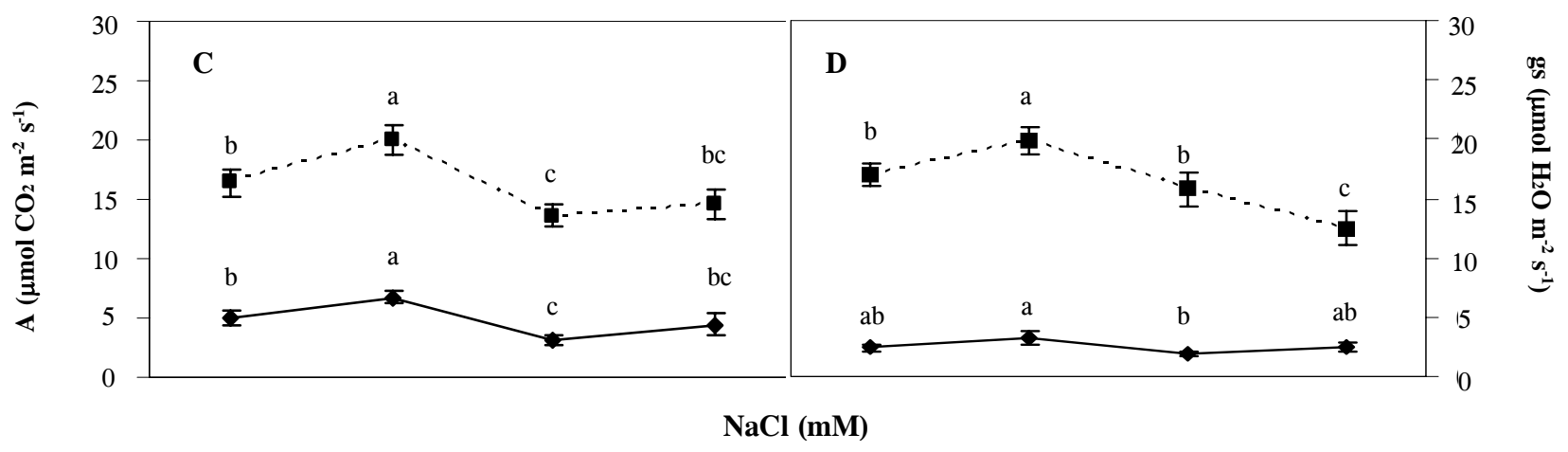

Figura 2. Efecto del $\mathrm{NaCl}$ en la tasa de asimilación de $\mathrm{CO}_{2}$ (--) (eje izquierdo) y conductancia estomática (-) (eje derecho) de Phaseolus después de 20 d de exposición a salinidad. Las letras sobre las barras indican diferencias significativas (Prueba DMS protegida de Fisher, P $\leq \mathbf{0 . 0 5}$ ). 

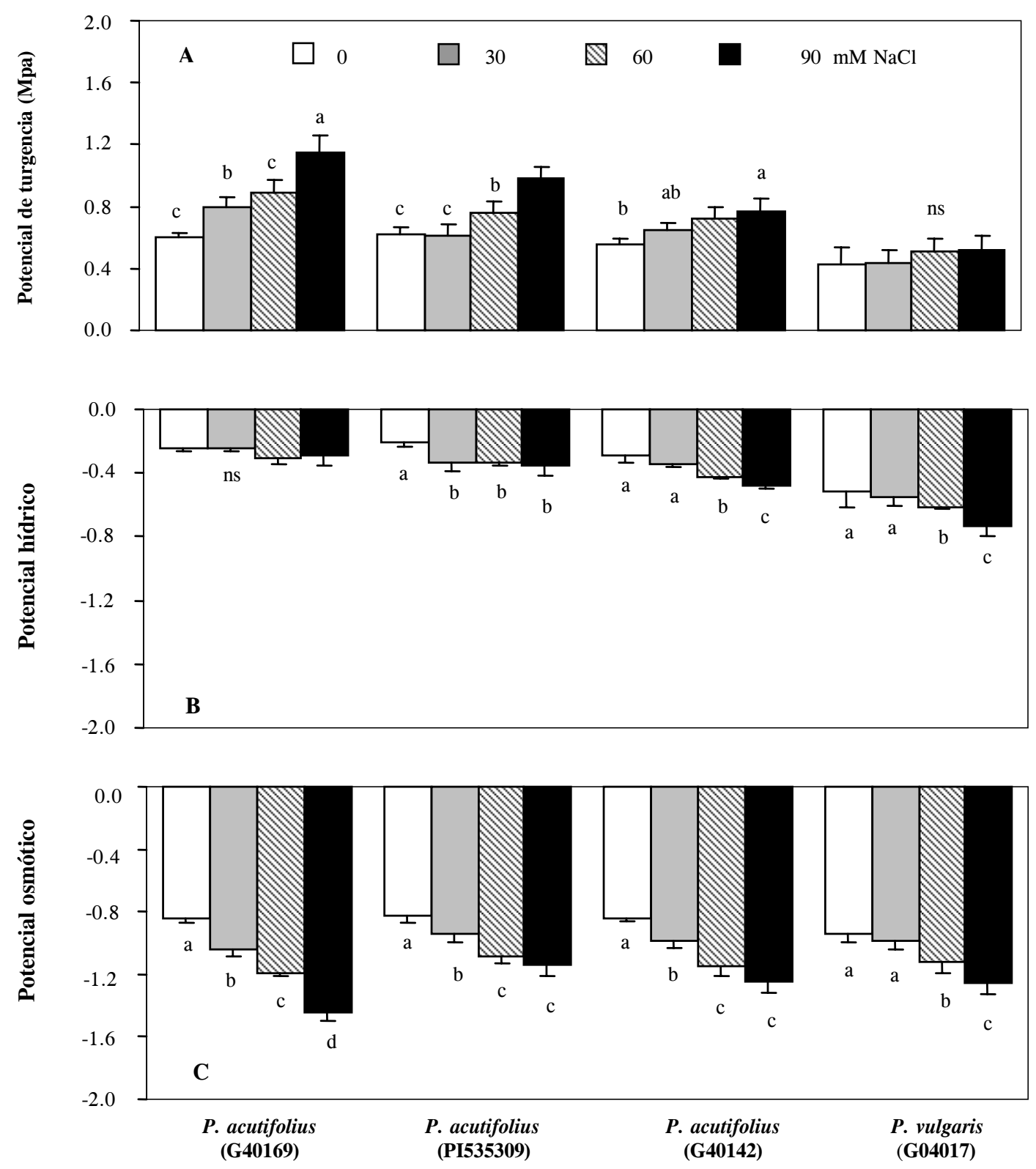

Figura 3. Efecto del $\mathrm{NaCl}$ en el potencial de turgencia (A), potencial hídrico (B) y potencial osmótico (C) (MPa) de Phaseolus después de 20 d de exposición a salinidad. Las letras sobre las barras indican diferencias significativas (Prueba DMS protegida de Fisher, P $\leq \mathbf{0 . 0 5}$ ).

\section{Carbohidratos totales}

El contenido de carbohidratos totales (CHO) de hoja, tallo y raíz se incrementó significativamente por efecto del $\mathrm{NaCl}$ (Cuadro 2). Asimismo, los cambios inducidos por la salinidad fueron dependientes de la especie. En todas las especies, excepto en $P$. filiformis, el contenido de CHO fue mayor en tallos que en hojas, a 60 y $90 \mathrm{mM}$ $\mathrm{NaCl}$ (Figuras 4A-B). El contenido de $\mathrm{CHO}$ de raíz, sin embargo, se mantuvo constante en todos los tratamientos salinos excepto en $P$. acutifolius G40142, en el que disminuyó significativamente en $90 \mathrm{mM} \mathrm{NaCl}$ (Figura 4C). $\mathrm{El}$ incremento del contenido de $\mathrm{CHO}$ totales en hojas y tallo con respecto al testigo osciló entre 65.8 y $130 \%$ y de 15 a $78 \%$, respectivamente. En particular, las especies de $P$. acutifolius (G40169) y P. acutifolius (G40142) alcanzaron la mayor concentración de CHO totales en tallo a $90 \mathrm{mM} \mathrm{NaCl}$, mientras que $P$. acutifolius $\mathrm{G} 40142$ y $P$. filiformis PI325687 presentaron la mayor acumulación en hojas (Figuras 4A-B). 


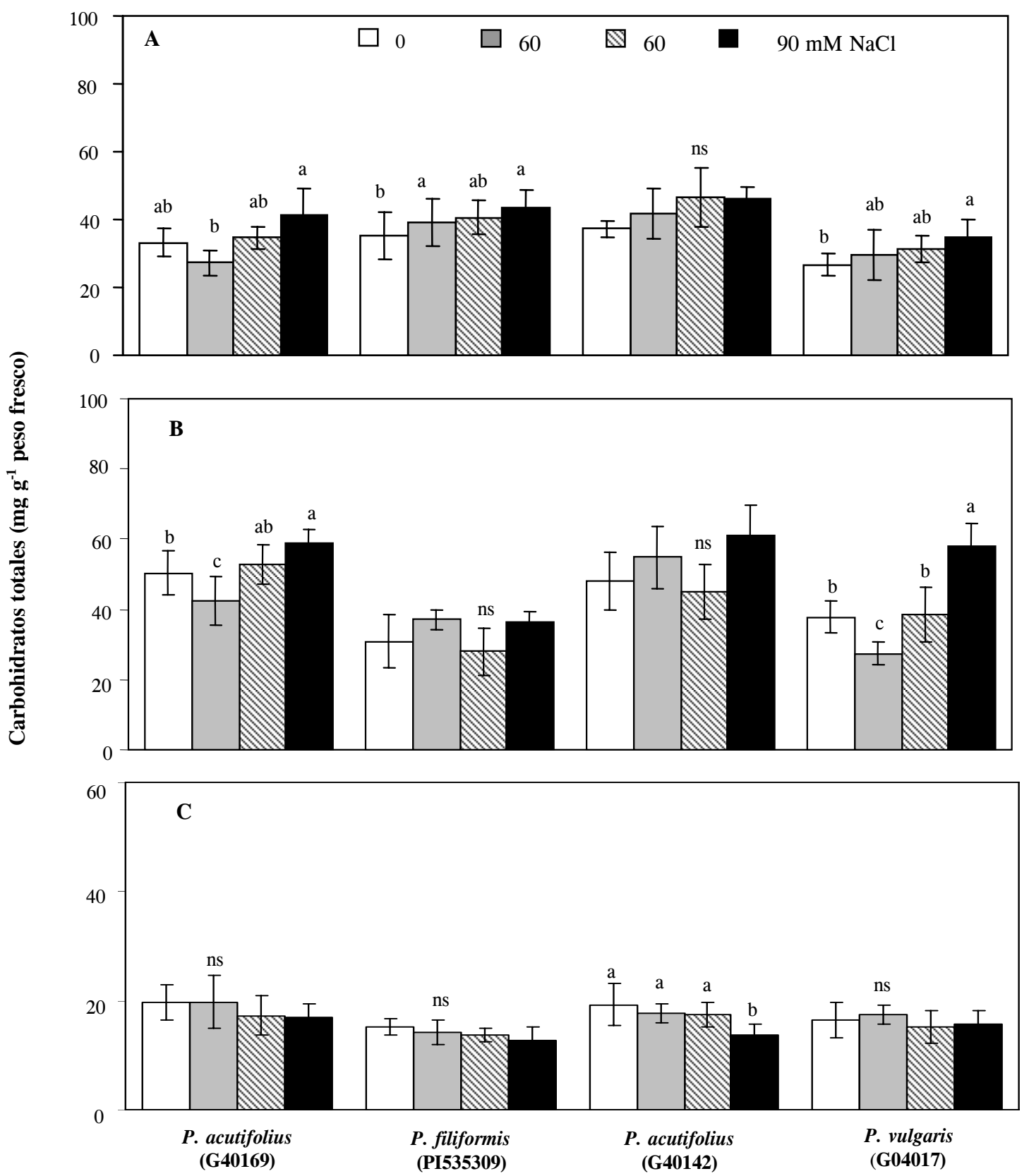

Figura 4. Efecto del agobio salino en el contenido de carbohidratos totales en hoja (A), tallo (B) y raíz (C) de Phaseolus spp., después de 20 d de exposición a salinidad. Las letras sobre las barras indican diferencias significativas (Prueba DMS protegida de Fisher, P $\leq \mathbf{0 . 0 5}$ ).

\section{DISCUSIÓN}

La disminución en la TCR puede atribuirse a cambios en la respuesta de la asimilación de $\mathrm{CO}_{2}$ (TAN) o en la razón de área foliar (RAF) (Bayuelo-Jiménez et al., 2003). Los resultados aquí obtenidos demuestran que la disminución de la TCR de todas las especies excepto de $P$. filiformis se relacionó con la TAN, lo que indica que la reducción del crecimiento en dichas especies se debió a una reducción de la eficiencia fotosintética del dosel (Figuras 1A-B). Asimismo, el análisis de regresión lineal entre TAN y TCR demostró que la menor eficiencia fotosintética del dosel repercutió en una más baja producción de materia seca en la especie silvestre $P$. acutifolius G40169 $\left(\mathrm{R}^{2}=0.93\right.$ a 0.97$)$ y en las especies cultivadas de P. acutifolius $\mathrm{G} 40142$ y P. vulgaris $\mathrm{G} 04017\left(\mathrm{R}^{2}=0.73\right.$ a 0.86). Resultados similares fueron reportados por Azevedo et al. (2004), Bayuelo-Jiménez et al. (2003) y Romero 
et al. (1994). La reducción de la TCR de las especies cultivadas también se asoció con la eficiencia en la producción de área foliar (RAF) (Figura 1C).

La disminución de RAF a mayor salinidad se relacionó significativamente con una reducción del incremento de materia seca por unidad de área (AFE). El coeficiente de determinación entre RAF y AFE $\left(\mathrm{R}^{2}=0.78\right.$ a 0.97$)$ confirman estos resultados. En $P$. filiformis, sin embargo, el AFE se mantuvo constante a 30 y $60 \mathrm{mM} \mathrm{NaCl}$ pero fue mayor que en el resto de los especies; es decir, esta especie desarrolló hojas más delgadas al elevarse la concentración salina (Figura 1E). De acuerdo con Baruch y Goldstein (1999), las especies que presentan una mayor AFE pueden tener una mayor TCR. En este estudio se demostró que $P$. filiformis no presenta una TCR mayor que el resto de los especies, pero su crecimiento no es afectado por las concentraciones salinas altas (Figura 1A). Lo anterior podría explicarse en términos de costos de carbono para construcción de estructuras foliares, que es menor para hojas delgadas (Dijkstra, 1989).

El agobio salino provocó diferencias significativas en el intercambio gaseoso y la conductancia estomática. El cierre estomático es un mecanismo efectivo para economizar la utilización de agua en condiciones de salinidad y evitar el daño por toxicidad iónica (Hasegawa et al., 2000). Sin embargo, una disminución en la conductancia estomática promueve simultáneamente una reducción en la tasa fotosintética (Brugnoli y Lauteri, 1991) y absorción de agua (Mencuccini y Grace, 1996). En el presente estudio, la tasa fotosintética disminuyó en todos los tratamientos salinos en comparación al testigo y durante los $20 \mathrm{~d}$ de duración del agobio, excepto en $P$. filiformis. De acuerdo con los resultados del análisis de regresión, la asimilación de $\mathrm{CO}_{2}$ depende de la conductancia estomática en todas las especies. Es pertinente destacar que $P$. filiformis PI535309 fue la única especie que presentó altas tasas de asimilación de $\mathrm{CO}_{2}$ y de conductancia estomática a 60 y $90 \mathrm{mM}$ de $\mathrm{NaCl}$, en comparación con el testigo.

Estos resultados demuestran que los procesos de fijación de $\mathrm{CO}_{2}$ y control estomático en $P$. filiformis son tolerantes a altas concentraciones de salinidad, y corroboran los resultados reportados por Bayuelo-Jiménez et al. (2003). Posiblemente el mecanismo de tolerancia a este nivel podría estar relacionado con diferencias en la densidad estomática y en la anatomía de la hoja, la respuesta de las células acompañantes, o bien a una combinación de estos factores (Plaut et al., 1990). Según Lynch et al. (1992), en condiciones ambientales de alta irradiancia o déficit hídrico en el suelo, una mayor densidad estomática en la lámina foliar favorece una mayor conductancia estomática y asimilación de $\mathrm{CO}_{2}$. Lo anterior es de especial interés en términos de mejoramiento genético de $P$. vulgaris, ya que la densidad estomática se encuentra relacionada con la eficiencia en el uso del agua. Asimismo, la eficiencia fotosintética de $P$. filiformis en condiciones de agobio salino $(80 \mathrm{mM} \mathrm{NaCl})$ se relaciona con un incremento en la tasa de absorción y acumulación de potasio en las hojas (Bayuelo-Jiménez et al., 2003). Esta especie mantiene concentraciones bajas de $\mathrm{Na}^{+}$y acumula mayor concentración de $\mathrm{K}^{+}$en la lámina foliar, lo que contribuye a una adecuada apertura estomática y eficiencia en el uso del agua.

$\mathrm{Al}$ igual que en la tasa de asimilación de $\mathrm{CO}_{2}$, el estado hídrico de la planta es sensible a la salinidad y, por tanto, es un factor determinante en su crecimiento (Munns, 2002). El análisis de las relaciones hídricas de Phaseolus mostró una respuesta a la salinidad común a las reportadas en otras especies (Lazcano-Ferrat y Lovatt, 1997). La disminución del potencial osmótico excedió a la del potencial hídrico, lo que permitió que el potencial de turgencia se mantuviera o incrementara en todas las especies. Esta respuesta celular conocida como ajuste osmótico es un mecanismo que confiere tolerancia a salinidad (Munns, 2002) y se encuentra estrechamente asociado con la regulación de la apertura estomática, actividad fotosintética y crecimiento vegetal. En la mayoría de plantas, la acumulación neta de iones inorgánicos o solutos orgánicos favorece el mantenimiento del potencial de turgencia (Hasegawa et al., 2000).

En este estudio se observó que la concentración de carbohidratos totales, particularmente de tallo y hojas, se incrementó a mayor concentración salina. En todas las especies estudiadas, la concentración de carbohidratos totales y el potencial osmótico tuvieron una correlación significativa en los tratamientos salinos $\left(R^{2}=0.42\right.$ a 0.80$)$, lo que indica que el incremento de carbohidratos contribuyó a la disminución del potencial osmótico de la planta. Es posible que la reducción de la fotosíntesis a mayores concentraciones de $\mathrm{NaCl}$ (Figura 2), haya alterado la cantidad de carbono fijado en el vástago y los azúcares transportados a la raíz (Azevedo et al., 2004). En la especie tolerante $P$. filiformis, sin embargo, hubo acumulación constante de carbohidratos en hojas, acumulación que no se interrumpió a concentraciones altas de $\mathrm{NaCl}$. Es evidente que esta respuesta esta relacionada con la mayor eficiencia fotosintética en condiciones de salinidad.

Las altas tasas de asimilación de $\mathrm{CO}_{2}$ y regulación de turgencia en $P$. filiformis se asocian con un mantenimiento constante de $\mathrm{K}^{+}$en las hojas y reducida absorción de $\mathrm{Na}^{+}$ al vástago (Bayuelo-Jiménez et al., 2003). Aunado a lo anterior, el incremento de carbohidratos en la raíz favoreció la absorción de agua, y esto se reflejó en el 
mantenimiento de potenciales hídricos favorables, como lo demuestra el coeficiente de correlación $\left(\mathrm{R}^{2}=0.61\right)$ (Figura 3), y que además se asoció con una adecuada tasa fotosintética y crecimiento $\left(\mathrm{R}^{2}=0.58\right)$. La tolerancia de esta especie podría relacionarse también con otros factores, como una menor inversión metabólica (costo energético) para la osmorregulación (ajuste osmótico) y una adecuada distribución de solutos orgánicos e inorgánicos entre las vacuolas y el citosol (Martínez-Ballesta et al., 2004).

Varios autores han señalado que la producción de solutos orgánicos es metabolitamente costosa y su contribución a la disminución del potencial osmótico celular es mínima y restrictiva para el crecimiento vegetal (Jouve et al., 2004; Arbona et al., 2005). En este estudio no se cuantificó el aporte de los carbohidratos en el ajuste osmótico pero fue evidente que el costo energético para su producción alteró el crecimiento, especialmente a mayor concentración salina. Una alternativa que contrarresta los costos energéticos de producción incluye la acumulación de iones del medio externo, aunque la acumulación excesiva de iones puede causar toxicidad y repercutir negativamente en las actividades bioquímicas de la célula (Munns, 2000). Las evidencias confirman que la acumulación de $\mathrm{Cl}^{-}, \mathrm{Na}^{+}$y $\mathrm{K}^{+}$en las hojas contribuyen significativamente $(65$ y $61 \%)$ con la reducción del potencial osmótico hasta -1.3 y $-1.62 \mathrm{MPa}$ a $40 \mathrm{mM} \mathrm{NaCl}$ y 80 $\mathrm{mM} \mathrm{NaCl}$, respectivamente y, consecuentemente con el mantenimiento de turgencia celular (Bayuelo-Jiménez et al., 2003) y la tolerancia al agobio salino.

\section{CONCLUSIONES}

Los resultados demuestran que $P$. filiformis es una especie tolerante a salinidad y que su variabilidad fisiológica contrasta con la del frijol cultivado ( $P$. vulgaris). Diversas características que confieren tolerancia a salinidad en esta especie silvestre (control estomático, eficiencia fotosintética y osmorregulación) podrían ser de valor como criterios de evaluación y selección en programas de mejoramiento de $P$. vulgaris cultivado.

\section{AGRADECIMIENTOS}

Al Programa de Profesores de Tiempo Completo, PROMEP-SEP, México (047/2003-2005) por el apoyo financiero otorgado para la realización del presente estudio.

\section{BIBLIOGRAFÍA}

Arbona V, A J Marco, D J Iglesias, M F López-Climent, M Talón, A Gómez-Cadenas (2005) Carbohydrate depletion in roots and leaves of salt-stressed potted Citrus clementina L. Plant Growth Reg. 46:153-160.

Azevedo Neto A, J T Prisco, J Enéas-Filho, C Feitosa de Lacerda, J Vieira Silva, P Alves da Costa, E Gomes-Filho (2004) Effects of salt stress on plant growth, stomatal response and solute accumulation of different maize genotypes. Brazil. J. Plant Physiol. 16:31-38.

Baruch Z, G Goldstein (1999) Leaf construction cost, nutrient concentration and net $\mathrm{CO}_{2}$ assimilation of native and invasive species in Hawaii. Oecologia 121:183-192.

Bayuelo-Jiménez J S, D G Debouck, J P Lynch (2003) Growth, gas exchange, water relations, and ion composition of Phaseolus species grown under saline conditions. Field Crop Res. 80:207-222.

Bayuelo-Jiménez J S, D G Debouck, J P Lynch (2002) Salinity tolerant of Phaseolus species during early vegetative growth. Crop Sci. 42:2184-2192.

Broughton W J, G Hernandez, M Blair, S Beebe, P Gepts, J Vanderleyden (2003) Beans (Phaseolus spp.) Model food legumes. Plant Soil 252:55-128.

Brugnoli E, M Lauteri (1991) Effects of salinity on stomatal conductance, photosynthetic capacity, and carbon isotope discrimination of salt-tolerant (Gossypium hirsutum L.) and salt-sensitive (Phaseolus vulgaris L.) $\mathrm{C}_{3}$ non halophytes. Plant Physiol. 95: 628-635.

Cramer G R, G J Alberico, C Schmidt (1994) Salt tolerance is not associated with the sodium accumulation of 2 maize hybrids. Aust. J. Plant Physiol. 21:675-692.

Dijkstra P (1989) Cause and effect of differences in specific leaf area. In: Causes and Consequences of Variation in Growth Rate and Productivity of Higher Plants. H Lambers, M L Cambridge, H Konings, T L Pons (eds). SPB Academic Publishing, The Hague, The Netherlands. pp:125-140.

Dubouis M, K A Guilles J K Hamilton, P A Rebers, S Smith (1956) Colorimetric method for determination of sugars and related substances. Analyt. Chem. 28:350-356.

Epstein E (1972) Mineral nutrition of plants: principles and perspectives. New York: J. Wiley \& Sons.

FAO (2005) Global Network on Integrated Soil Management for Sustainable Use of Salt-affected Soils. Rome Italy: FAO Land and Plant Nutrition Management Service. http://www.fao.org/ag/agl/agll/spush (Febrero 28, 2008).

Hasegawa P M, R A Bressan, J K Zhu, H J Bohner (2000) Plant cellular and molecular responses to high salinity. Annu. Rev. Plant Physiol. Plant Mol. Biol. 51:463-499.

Hunt R (1990). Basic Growth Analysis. Plant Growth Analysis for Beginners. Unwin Hyman. London. 112 p.

Jouve L, L Hoffmann J F Hausman (2004) Polyamine, carbohydrate, and proline content changes during salt stress exposure of Aspen (Populus tremula L.): Involvement of oxidation and osmoregulation metabolism. Plant Biol. 6:74-80.

Läuchli A (1984) Salt exclusion: an adaptation of legumes for crops and pastures under saline conditions. In: Saline Tolerance in Plants: Strategies for Crop Improvement. R C Staples, G H Toenniessen (eds). Wiley, New York. pp:171-178.

Lazcano-Ferrat I, C L Lovatt (1997) Effect of salinity on arginine biosynthesis in leaves of $P$. vulgaris $\mathrm{L}$. and $P$. acutifolius A. Gray. Crop Sci. 37:469-475.

Lynch J, A González, J M Tohme, J A García (1992) Variation in characters related to leaf photosynthesis in wild bean populations. Crop Sci. 32:633-640.

Martínez-Ballesta MC, V Martínez, M Carvajal (2004) Osmotic adjustment, water relations and gas exchange in pepper plants under $\mathrm{NaCl}$ or KCl. Env. Exp. Bot. 52:161-174.

Mencuccinni M, J Grace (1996) Development patterns of above ground hydraulic conductance in a Scots pine (Pinus silvestris L.) age sequence. Plant Cell Env. 19:939-948. 
Munns R (2002) Comparative physiology of salt and water stress. Plant Cell Env. 28:239-250.

Nobel P S (1991) Physicochemical and Environmental Plant Physiology. 2nd ed. New York: Academic Press. 555 p.

Ojeda-Real L, R Cárdenas-Navarro, P Lobit, O Grageda-Cabrera, E Valencia-Cantero, L Macías-Rodríguez (2008) Efecto de la nutrición nítrica y sistemas de riego en el sabor de la fresa. Rev. Chapingo S. Hort. 14:61-70.

Plaut Z, M C Grieve, E V Maas (1990) Salinity effects on $\mathrm{CO}_{2}$ assimilation and diffusive conductance of cowpea leaves. Physiol. Plant. 79:31-38.

Romero J M, T Marañón, J M Murillo (1994) Long-term responses of Melilotus segetalis to salinity. I. Growth and partitioning. Plan Cell Env. 7:1243-1248.

Ruiz V V M, M Ortega E, V A González, J J Martínez H, J L Rone P (1997) Respuesta del frijol a la salinidad sulfático- clorhídrica I. Producción de biomasa y rendimiento. Rev. Fitotec. Mex. 20:13-27.

SAS Institute (1995). SAS User's Guide: Statistics. 5th ed. Cary, NC: SAS Institute.

Serraj R, T R Sinclair (2002) Osmolyte accumulation; can it really help increase crop yield under drought conditions? Plant Cell Env. 25:333-341.

Scholander P F, H T Hammel, E D Bradstress, E A Hemingsen (1965) Sap pressure in vascular plants. Science 148:339-346.

Stepien P, G Klobus (2006) Water relations and photosynthesis in $\mathrm{Cu}$ cumis sativus L. leaves under salt stress. Biol. Plant. 50:610616.

Yeo A R, S P Caporn, T J Flores (1985) The effect of salinity upon photosynthesis in rice (Oryza sativa L.): Gas exchange by individual leaves in relation to their salt content. J. Exp. Bot. 36:1240-1248 